\title{
Endoscopic submucosal dissection of early gastric cancer via inverted overtube in a patient with situs inversus totalis:
} a case report

\section{다(1) $(9)$}

\author{
Authors \\ Institutions \\ 1 Division of Endoscopy, Shimane Prefectural Central \\ Hospital, Shimane, Japan \\ 2 Division of Gastroenterology, Shimane Prefectural \\ Central Hospital, Shimane, Japan \\ 3 Division of Liver, Shimane Prefectural Central Hospital, \\ Shimane, Japan \\ 4 Division of Pathology, Shimane Prefectural Central \\ Hospital, Shimane, Japan \\ 5 Division of Endoscopy, Kaken Hospital, International \\ University of Health and Welfare, Chiba, Japan
}

Youichi Miyaoka', Shinsuke Suemitsu' ${ }^{2}$, Aya Fujiwara², Satoshi Kotani ${ }^{1}$, Kosuke Tsukano ${ }^{2}$, Satoshi Yamanouchi², Ryusaku Kusunoki ${ }^{2}$, Tatsuya Miyake ${ }^{3}$, Hirofumi Fujishiro ${ }^{2}$, Naruaki Kohge ${ }^{2}$, Tomohiko Yamamoto ${ }^{4}$, Yuji Amano ${ }^{5}$

submitted 25.10.2017

accepted after revision 17.1.2018

Bibliography

DOI https://doi.org/10.1055/a-0581-7101 |

Endoscopy International Open 2018; 06: E625-E629

(c) Georg Thieme Verlag KG Stuttgart · New York

ISSN 2364-3722
Corresponding author

Youichi Miyaoka, MD, PhD, Division of Endoscopy, Shimane Prefectural Central Hospital, 4-1-1 Himebara, Izumo 693-

8555, Shimane, Japan

Fax: +81-0853-21-2197

miyaoka@spch.izumo.shimane.jp

\section{ABSTRACT}

Background and study aims A 72-year-old man with complete situs inversus presented with early gastric cancer on the lesser curvature wall of the antrum of the stomach. Endoscopic submucosal dissection (ESD) was selected as a treatment. When the patient was positioned in the left decubitus position, the lesion was hidden by blood and gastric fluid because it was located on the gravitational side. Therefore, we decided to perform ESD with the patient in the right lateral decubitus position and use an inverted overtube, which provided a good endoscopic view without the need to rearrange the endoscopist, assistants, or endoscopic system. ESD was safe and feasible using the inverted overtube.

\section{Introduction}

Situs inversus totalis (SIT) is defined as the complete mirror-image transposition of the thoracic and abdominal viscera. It is a rare congenital anomaly with an incidence of 1:6200-6600 [1]. Although this condition does not affect normal health or longevity, advanced recognition is important for management of many diseases and procedures, including endoscopic and surgical interventions [2]. Endoscopic procedures can be particularly difficult due to the inverted position of the stomach.

Endoscopic mucosal dissection (ESD) has been widely accepted as a treatment for early-stage gastric cancers [3]. However, ESD is a delicate procedure that carries risk of complications such as bleeding and perforation, and particular attention must be paid in patients with SIT.

Previous literature regarding ESD for gastric cancer in patients with SIT is limited [4], although there are some reports of surgical intervention in these cases $[2,4]$. Herein we report a patient with SIT and gastric cancer who underwent ESD using an inverted overtube. The overtube consists of a linear part and a curved part and allows endoscopy to be performed with the patient in the right lateral decubitus position [5]. To the best of our knowledge, this is the first description of gastric ESD using an inverted overtube in a patient with SIT.

\section{Case report}

A 72-year-old man visited our hospital for evaluation of a gastric lesion identified on screening esophagogastroduodenoscopy (EGD) at an outside hospital. No biopsies had been performed. He had been diagnosed with SIT several decades earlier. He had multiple medical comorbidities, including sick sinus syndrome (SSS), atrial fibrillation, angina, cerebral infarction sequelae, hypertension, and glaucoma. He had undergone Heli- 

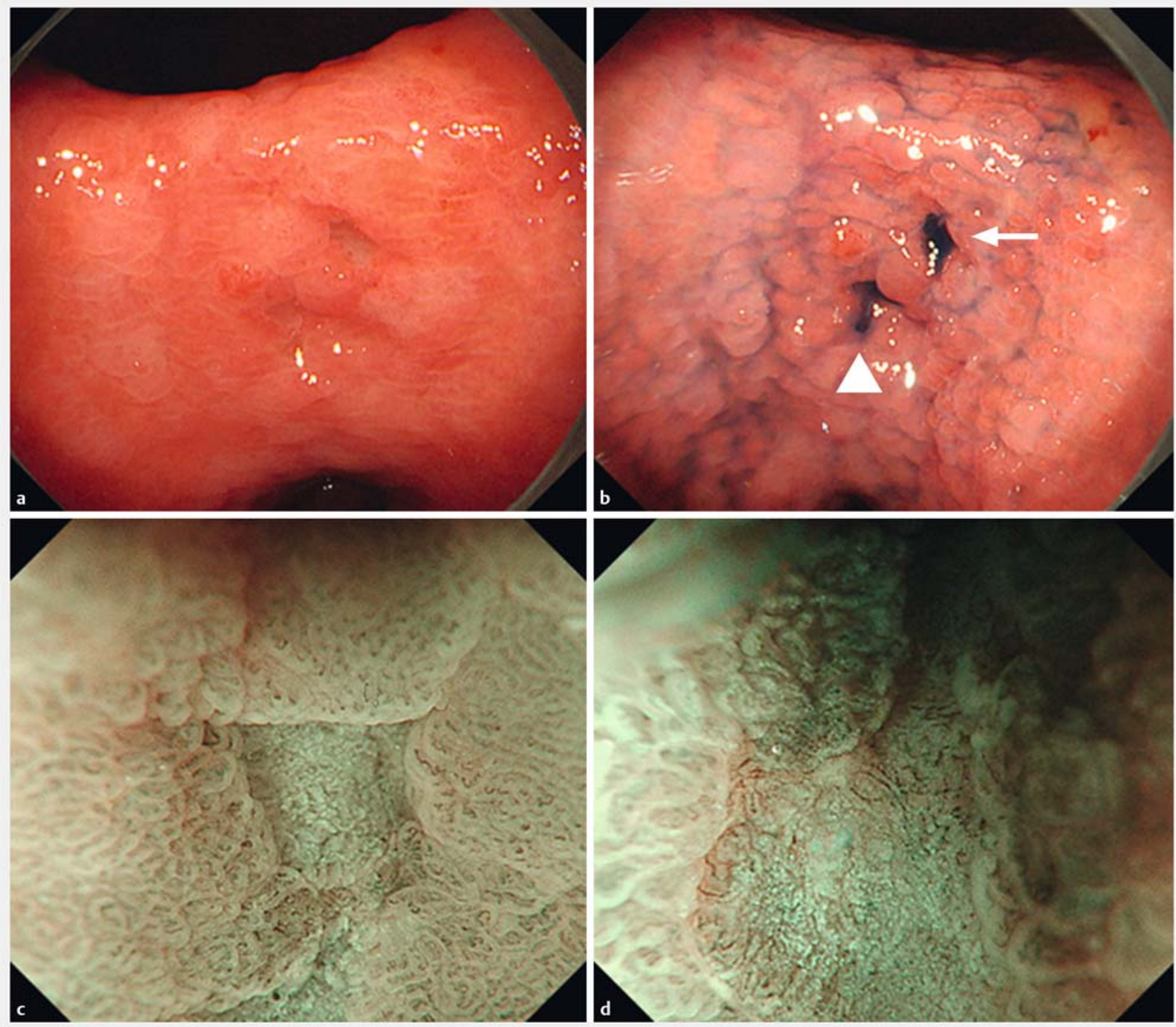

- Fig. 1 a White light endoscopy revealed two irregularly shaped depressed lesions with a circumferential flat elevated area on the lesser curvature wall of the antrum of the stomach. $\mathbf{b}$ Chromoendoscopy with indigo carmine clarified the depressed lesions (white arrow and white

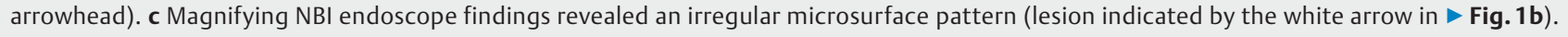
d Magnifying NBI endoscope findings revealed irregular microsurface and microvascular patterns (lesion indicated by the white arrowhead in Fig.1b).

cobacter pylori eradication therapy about 10 years prior. His medications included two kinds of antithrombotic drugs (antiplatelet agent: aspirin and anticoagulant agent: warfarin) and he had a cardiac pacemaker for SSS. Blood tests revealed an elevated PT-INR (2.57) due to treatment with warfarin.

EGD was performed using a magnifying endoscope (GIFH260Z, Olympus, Tokyo, Japan). At the lesser curvature of the antrum, white light endoscopy revealed a slightly elevated lesion which was covered with non-neoplastic gastric mucosa, with two irregular depression ( $\triangleright$ Fig.1a). Chromoendoscopy with indigo carmine clarified the shape of the two depressed areas ( $\triangleright$ Fig.1b). Magnifying narrow band imaging (NBI) endoscopy showed irregular microsurface and microvascular patterns, suggesting a carcinomatous lesion, in each depressed area and the surrounding mucosal surface showed a pattern consistent with normal pyloric gland mucosa ( $\downarrow$ Fig. 1c,d). Biopsy specimens taken from the depressed areas were diagnosed as indefinite for neoplasia (Vienna classification category 2). However, early-stage gastric cancer was strongly suspected due to the NBI magnifying endoscopic findings and ESD was thus performed.

The target lesions were located on the side of the stomach subjected to gravity while in the left lateral decubitus position, therefore the therapeutic view-field might be obscured by gastric fluid and blood. Most endoscopists perform ESD in the left lateral decubitus position; the right lateral decubitus position is 


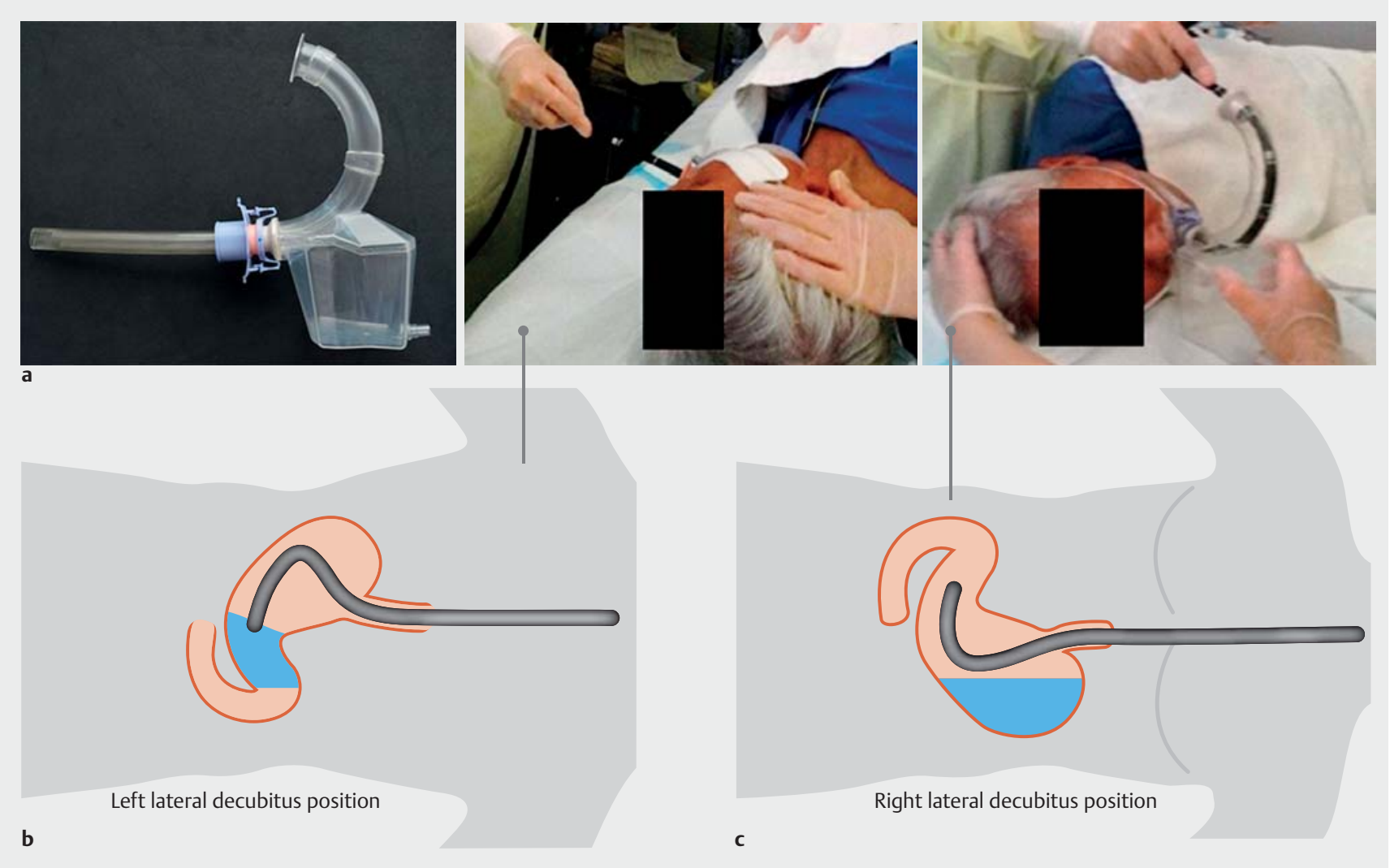

- Fig. 2 a The inverted overtube (ENDRESCUE, 13B1X00085000109; TOP Corporation, Tokyo, Japan). b In this patient with SIT, the lesion was located at the lesser curvature of the gastric antrum. Because the lesion was located on the gravity side in the left lateral decubitus position, it was masked by blood and gastric fluid. $\mathbf{c}$ The patient was rolled to the right lateral decubitus position. With use of an inverted overtube, a good endoscopic view was obtained.
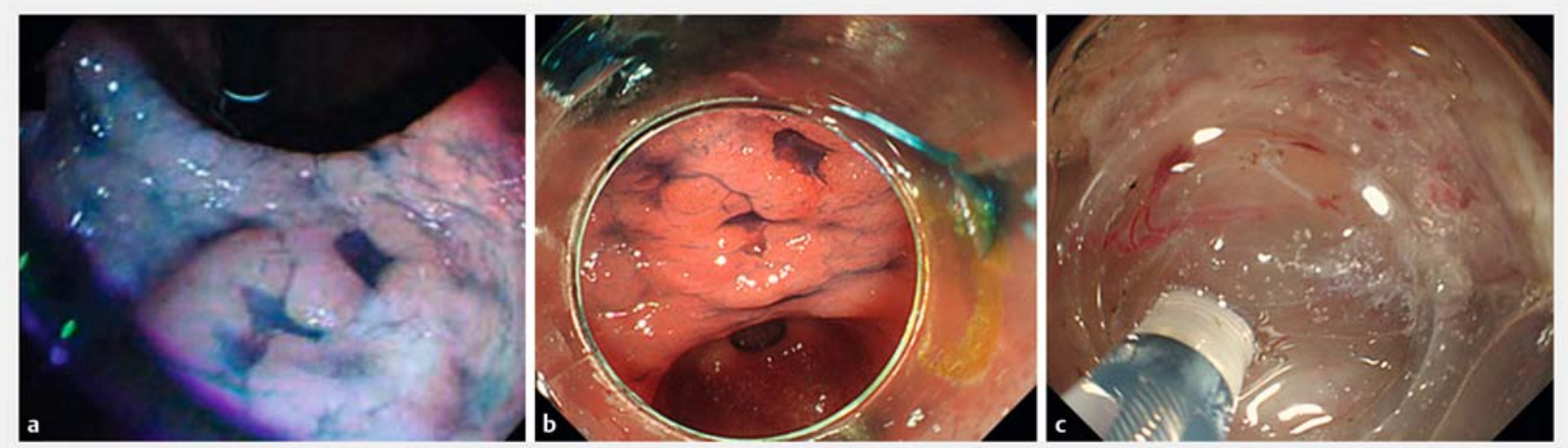

- Fig. 3 a Chromoendoscopic image with indigo carmine in the left lateral decubitus position. The caudal end of the lesion could not be approached in this position. $\mathbf{b}$ Chromoendoscopic image with indigo carmine in the right lateral decubitus position. A good endoscopic view was obtained. c A good endoscopic view of the submucosal layer was obtained and submucosal dissection was safely performed.

more technically difficult. Therefore, we used an inverted overtube (ENDRESCUE, 13B1X00085000109; TOP Corporation, Tokyo, Japan) to overcome the particular difficulties of this case [5] ( $\triangleright$ Fig. 2a). First, the endoscope was inserted while the patient was in the left lateral decubitus position and the linear part of the overtube was introduced ( $\boldsymbol{\nabla}$ Fig. 2 b). After removing the endoscope, the patient was moved to the right lateral decu- bitus position and the curved part of the overtube was connected. The endoscope was reinserted and ESD was performed ( $\triangleright$ Fig.2c). In the left lateral decubitus position, the caudal end of the lesion was hard to observe and the gastric fluid easily interfered with the endoscopic view, so the lesion was impossible to fully evaluate ( $\triangleright$ Fig. 3a). On the other hand, in the right lateral decubitus position, a much clearer endoscopic view was 

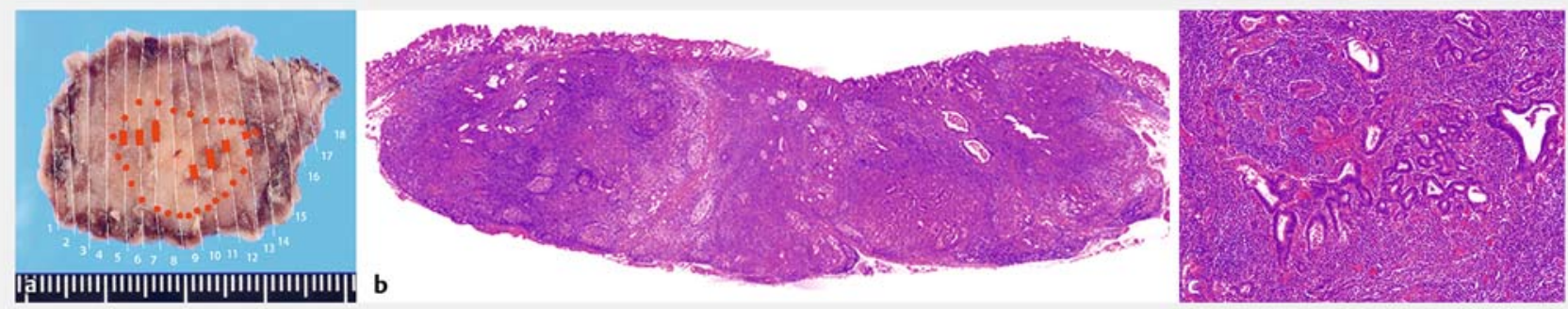

- Fig. 4 Pathological findings. a Distribution map of the tumor. Red dots indicate the range of the cancer underlying the mucosal layer and the red line indicates the exposed cancer on the mucosal surface. b Loupe images of slice No.10 (white line on the map). The submucosal layer was fully occupied by tumor tissue. c Histopathology revealed a tubular adenocarcinoma with lymphoid stroma.

obtained ( $>$ Fig. 3b, c). Furthermore, the inverted overtube allowed all the endoscopic devices, including the light source, to be set in their usual positions. With this method, the lesion was completely removed without complication.

The resected specimen was $41 \times 24 \mathrm{~mm}$ in diameter, and included a flat elevated lesion $(20 \times 13 \mathrm{~mm})$ ( $\mathbf{F i g . 4 a )}$. Histopathologic features of the specimen are shown in $>$ Fig. $\mathbf{4 b}$ and

- Fig. 4c. The histopathology revealed a tubular adenocarcinoma with lymphoid stroma. In situ hybridization was negative for Epstein-Barr virus-encoded small RNA (EBER). Carcinoma cells invaded the submucosa to approximately $3300 \mu \mathrm{m}$ without lymphatic or venous invasion. Although for the most part the lesion was covered with non-neoplastic epithelium, a small amount of carcinoma was exposed to the surface in the depressed areas.

Although the patient was proposed to undergo gastric resection with lymphadenectomy since the resection was regarded as non-curative, he insisted on refusing the surgery. He was followed for 42 months in the outpatient department and has not had a cancer recurrence.

\section{Discussion}

To the best of our knowledge, this is the first report of an early gastric carcinoma in a patient with SIT that was successfully treated with ESD using an inverted overtube. SIT is a relatively rare condition caused by an autosomal-recessive congenital defect in which an abdominal and/or thoracic organ is positioned in the "mirror image" of the normal position in the sagittal plane $[2,4,6]$. Previous reports of gastric cancer surgery in patients with SIT have described surgical difficulties due to anatomic anomalies, including differences in the perigastric major vessels [2, 4, 6, 7]. Endoscopic surgery is also more difficult because of differences in gravitational effects on the stomach, which restrict endoscopic manipulations.

ESD for early gastric cancer is performed around the world [3]. To safely perform ESD, it is important to maintain a good endoscopic view wherein the lesion is not submerged in liquid such as injected water or blood. Most endoscopists prefer the patient in the left lateral decubitus position, which allows for a clear endoscopic view. If persistent bleeding occurs, lesions may be hidden by clots. The maneuverability of the endoscope in the right decubitus position in SIT patients is similar to that in the left decubitus position in non-SIT patients. Mori H. et al. reported the efficacy of a newly developed inverted overtube to shorten hemostatic time and obtain a clear endoscopic view in emergency endoscopic hemostasis [5]. In the current case, the lesion was masked by blood and residual gastric fluid when the patient was in the left lateral decubitus position because the lesion was located on the gravitational side. Therefore, good countertraction could not be obtained. After rotation to the right lateral decubitus position and with use of an inverted overtube, a clearer endoscopic view and good traction were obtained without the need for other methods such as dental floss clip traction [8] or double-endoscope ESD [9].

The overtube device is easy to install and can be used by both trainees and experts. However, some difficulties remain. Because the endoscope passes through the curved portion of the overtube, a slight interference between the overtube and the endoscope occurs. This can be dealt with by injecting lubricating jelly into the overtube. Furthermore, the inverted overtube may move occasionally due to movement of the endoscope, and must be fixed by hand.

\section{Conclusion}

In conclusion, this is the first report of ESD for gastric cancer in a patient with SIT using an inverted overtube with the patient in the right decubitus position. This method was safe and feasible and provided a good endoscopic view with appropriate traction while allowing for standard endoscopic equipment arrangement.

\section{Competing interests}

None

References

[1] Blegen HM. Surgery in situs inversus. Ann Surg 1949; 129: 244-259

[2] Benjelloun el B, Zahid FE, Ousadden A et al. A case of gastric cancer associated to situs inversus totalis. Cases J 2008; 1: 391 
[3] Ono H, Yao K, Fujishiro M et al. Guidelines for endoscopic submucosal dissection and endoscopic mucosal resection for early gastric cancer. Digestive Endoscopy 2016; 28: 3-15

[4] Sumi Y, Maehara R, Matsuda Y et al. Laparoscopy-assisted distal gastrectomy in a patient with situs inversus totalis. JSLS 2014; 18: 314 318

[5] Mori H, Kobara H, Fujihara S et al. Accurate hemostasis with a new endoscopic overtube for emergency endoscopy. World J Gastroenterol 2013; 19: $2723-2726$

[6] Morimoto M, Hayakawa T, Kitagami H et al. Laparoscopic-assisted total gastrectomy for early gastric cancer with situs inversus totalis: report of a first case. BMC Surgery 2015; 15: 75
[7] Suh B]. A Case of Gastric Cancer with Situs Inversus Totalis. Case Rep Oncol 2017; 10: $130-135$

[8] Yoshida M, Takizawa K, Ono H et al. Efficacy of endoscopic submucosal dissection with dental floss clip traction for gastric epithelial neoplasia: a pilot study (with video). Surg Endosc 2016; 30: 3100 - 3106

[9] Higuchi K, Tanabe S, Azuma M et al. Double-endoscope endoscopic submucosal dissection for the treatment of early gastric cancer accompanied by an ulcer scar (with video). Gastrointest Endosc 2013; 78: $266-273$ 